International Journal of Computer Networks \& Communications (IJCNC) Vol.9, No.1, January 2017

\title{
Position Based AdAPtive Routing For VANETS
}

\author{
Ajay Guleria ${ }^{1}$ and Kuldeep Singh ${ }^{2}$ \\ ${ }^{1}$ Computer Centre, Panjab University, Chandigarh, India \\ ${ }^{2}$ Computer Science \& Application Department, Panjab University, Chandigarh, India
}

\begin{abstract}
Routing plays a very significant role in multi hop data dissemination in Vehicular Ad-Hoc Networks (VANETs). Wehave proposed a Position based Adaptive Routing (PAR) protocol which is scalable for different network densities in VANETs. This scheme uses Preferred Group Broadcasting (PGB) for route discovery. In this mode, after broadcasting the request for route discovery the source node starts listening to the channel. If the packet is not further rebroadcasted by any neighbor in a set timeout, then it repeats the broadcast. This process is repeated until the request reaches the destination. The destination keeps on accumulating route requests coming from different paths until predefined time. It then chooses the least cost path as route reply. It uses the set of traversed anchors for sending the unicast route reply to the source node. PAR uses Advance Greedy Forwarding (AGF) for data forwarding and greedily forwards the data packet to the next anchor towards destination node. It switches to carry and forward mode once it finds partitions in the network. The intermediate vehicle buffers the packet until next junction and switches back to position based scheme and greedily forwards to next node in range which is closest to the destination. To have an end to end connectedpath, it uses guards to guard anchors tied to different junction and geographical locations in the network. The algorithm is scalable and exploits advantages of existing techniques already developed for specific scenarios in VANET. Results show that the service ratio and packet delay of PAR are higher than its counterparts.
\end{abstract}

\section{KEYWORDS}

$P A R, P G B, A G F$

\section{INTRODUCTION}

The coverage of road side unit (RSU) is limited by its radio range, so vehicles entering in its range only can seek or upload data. The data disseminated by road side unit may be beneficial to the other vehicles driving miles away. People driving in a specific area may be interested to reserve a parking place, book an accommodation, finding out petrol prices, find out the best dining outlet in the area. There may be distant vehicles, who have acquired some urgent information like accident on a particular road segment, traffic congestion or other safety related information to be uploaded on RSU server. Data deliveries for all these applications are feasible by wireless tech-

DOI: 10.5121/ijcnc.2017.9105 
nologies like 4G, 3G, etc., but the cost of providing them through these platforms is very costly. These applications are also feasible by multi-hop data dissemination in VANET. In multi-hop data dissemination vehicles use intermediate vehicles as relays to reach other vehicles and the road side units.

Multi-hop communication in VANET does add its own complexities into the network. These networks are highly mobile, and have intermittent connectivity issues. Urban area observes heavy network density during peak hours, while rural areas or highways generally have sparse traffic during night time. Designing a data dissemination strategy which can handle these two extreme situations is a challenging task. On one hand, in highly dense urban scenario selection of intermediate nodes for multi-hop data dissemination is based on reduction of too much control packets from wireless channel, while on the other side in case of sparse network when no neighbor exists in its range towards destination, carry and forward of data packets by vehicles is the only way out [5].

An efficient and scalable routing is one of the key aspect of a successful multi-hop communication algorithm for VANET. Research in past [1-3, 10-12] has shown that existing routing algorithms for mobile ad hoc networks (MANETs) are not able to adapt to unique requirements of vehicular ad hoc networks. These new technical challenges include highly unstable network topology, scalability issues as network may be very dense or have partitions. So the routing solutions designed for VANET must address all these new requirements in addition to the basic design issues. However, few additional information is available in vehicular ad hoc networks like predicted mobility pattern as vehicle plies on certain road only, trajectory of routing path can be precomputed, vehicles use stored digital maps, they use navigation software. All this information can be used to make better routing decisions.

Routing algorithms used in vehicular networks can be divided into three categories: (1) proactive, (2) reactive, and (3) geographical. In case of proactive algorithms, nodes taking part in routing maintain a routing table, which is built by exchanging link state messages with other nodes of the network. Thus, routes are computed and maintained in advance, even if there is no data for exchange in the network. The performance of these protocols degrades when the traffic density becomes very large as nodes exchange too much control information and leaves very less channel for data transmission. The second approach, reactive routing only searches for a route when it is needed. The sending node does not know in advance how to reach the destination. Route discovery is done on demand. This requires flooding of route requests in the network which usually increases the end to end delay as the source has to wait for more time before the routing path is established. On demand reactive protocols use broadcasting for the route discovery, so control packets may flood and congest the channel. Moreover, there may be a case when the intermediate vehicle or source vehicle may not find any neighbor for relaying request. Geographic routing generally also works on demand. However, the technique used by them is very different from on demand routing protocols. Geographic routing protocols do not use the topology of the network for routing information rather these algorithms uses geographic coordinates of nodes and take routing decisions hop-by-hop in a per-packet basis. Each relay selects its next hop based on its position, its neighbors' positions, and the position of the destination. These algorithms also suffer due to radio obstacles or intermediate nodes might find them in local maximum $[2,4]$. Special requirements, like highly mobile network topology, varying densities and intermittent connectivi- 
ty makealready existing routing protocols of the above categories unsuitable for all kind of scenarios possible in VANET. So the better idea is to make an adaptive routing protocol which adapts according to the situations.

This paper discusses a new routing strategy for the multi-hop efficient data dissemination in vehicular ad hoc networks. The proposed Position based Adaptive Routing (PAR) scheme is a position based algorithm which uses Preferred Group Broadcasting (PGB) for route discovery [3]. It switches to carry and forward [5] until next junction, once theintermediate node fails to find any vehicle to handover information towards destination. The scheme uses anchors to record the list of junctions traversed by the packet on its way to the destination. For path maintenance it uses the concept of guards [6].

Our work is aimed to provide an adaptive routing scheme which switches between geographical and carry and a forward mode based on actual traffic scenario and is scalable for all kinds of network densities in VANET. The rest of this chapter is organized as follows. Section 2 describes preliminaries and system model. Section 3 describes PAR routing scheme, and Section 4 evaluates the performance of the proposed scheme. Concluding remarks are given in Section 5.

\section{Preliminaries and System Model}

\subsection{Preliminary}

Vehicular ad hoc network's predictive mobility patterns and highly dynamic topology pose challenging demands on routing methods to deal with such environment $[2,3,7,8]$. These demands make routing algorithms to use positions of nodes in order to provide successful communication from the source to the destination. Most of proactive and reactive routing protocols do not perform well in these kinds of environments $[1,2,6,8]$. So position based routing in which geographical positions of nodes are used to perform data routing is preferred [2, 4]. In the position based routing approaches an intermediate node forwards a packet to the direct neighbor,who is closest to the geographic position of the destination, this is called Greedy Forwarding [2]. To participate in position based routing, each node has to be aware of:

- $\quad$ Its own position.

- $\quad$ Position of its direct neighbors.

- $\quad$ Position of the destination.

A node determines its own position by using GPS, the position of the neighbors is received through one hop beacon messages transmitted periodically by all nodes and the position of the final destination is provided by a location service $[2,4]$. Greedy forwarding uses only local information to select the next hop towards the destination, so it may end up in local optimum [2] i.e., no neighbor exists which is closer to the destination than the intermediate node itself. In order to escape from a local optimum a repair strategy is used. Greedy Perimeter Coordinator Routing (GPCR) is a solution for this problem [2, 4, 6]. This algorithm uses restrictive greedy routing along with perimeter coordinate routing. Another case can arise when traffic is sparse and inter- 
mediate node is not able to find any neighbor to hand over the data packet towards thedestination, in such cases carry and forward mechanism is deployed [5].

\subsection{Assumptions and Model}

The following assumptions are made:

- $\quad$ Vehicles are equipped with wireless transceivers.

- $\quad$ Vehicles are equipped with dedicated short-range communications radio modules as well as Global Positioning System (GPS), by which vehicles can determine their geographical location.

- Vehicles find location of the destination through location service.

- $\quad$ Vehicles find neighbors through one hop beacon messages.

- Vehicles place packet delivery information such as packet source id, its vector velocity, packet generation time, destination location, expiration time, etc., in the packet header.

- $\quad$ Vehicles are preloaded with digital maps, which provide street maps and traffic statistics at different times of the day in the area.

- $\quad$ The communication paths are available via the ad hoc network when there is no other communication infrastructure.

- $\quad$ Battery power and processing power of the vehicular node is not the limiting factor for the design.

The model is described in the following section:

Each vehicle in VANET is equipped with On Board Unit (OBU) which is transceiver with computational power and omni-directional antenna. Vehicles communicate witheach other through a short range wireless channel. OBU on the vehicles is capable of calculating average speed of vehicles. Vehicles can request the desired data from theother vehicles or from a nearby RSU. We have assumed a road network of multiple segments forming diagonal streets in city or rural area. A typical network is shown in Figure 1. As shown, source vehicle and destination vehicle are moving in different road segments. Source vehicle uses theintermediate vehicles for sending information to the destination vehicle. As shown in Figure 1, there are multiple paths possible from the source vehicle to the destination vehicle. The proposed algorithm selects the most populated path to send information to the destination.

We also assumed that the arrival rate of avehicle in the particular road segment $\mathrm{R}_{\mathrm{ij}}$ follows Poisson distribution with mean arrival rate $\lambda$ vehicles per second. So the inter-arrival times of vehicles are exponentially distributed with expected inter arrival time equal to $1 / \lambda$. Let the vehicle density of the road segment $R_{\mathrm{ij}}$ be $\rho_{\mathrm{ij}}$. We have also assumed that there is no congestion in road segment $R_{\mathrm{ij}}$ and vehicles travel at free flow at speed $v$ meters per second. Then by assuming an $\mathrm{M} / \mathrm{M} / 1$ queue model for a free flow road, we have arrival rate $\lambda=\rho_{i j} \times v$. Let each vehicle has a radio range $T_{x}$. Two vehicles are connected if the physical distance between two vehicles is less than $T_{x}$. The inter-arrival time between two vehicles $i$ and $i+1$ is denoted by the random variable $T_{i}$. The density function of $\mathrm{T}_{\mathrm{i}}$ is given by:

$f_{n}(t)=\lambda e^{-\lambda t},(t \geq 0)$ 
Let the inter vehicle distance between vehicle $i$ and $i+1$ be denoted by $D_{i}$. When using a free flow for road segment $\mathrm{R}_{\mathrm{ij}}$, and assuming $\mathrm{v}$ as the nominal speed of the vehicles on this segment we have $D_{i}=v \times T_{i}$.

$f_{D(d)}=\rho_{i j} \times e^{-\rho_{i j}} d,(d \geq 0)$

The spatial distribution of vehicles on the road segment is also a Poisson process. The probability $P_{d}$ that any moving vehicle will find at least one vehicle in its transmission range $T_{x}$ for transmitting packet is given by:

$P_{d=1-e^{\rho_{i j T x}}}$

So, the probability of not finding any vehicle in its radio range is when $P_{d}=e^{-\rho_{i j T^{x}}}$. So in that case vehicle will use carry and forward method and when $P_{d=1-e^{\rho_{i j} T_{x}}}$ it finds another vehicle which is selected as next hop to forward data packet to the destination.

The proposed PAR works on following principles:

Use restive greedy forwarding to forward a packet to the direct neighbor which is closest to the geographic position of the destination. Transmit through wireless channels, as much as possible [5].

a) In case of intermediate node getting stuck up in local maximum or in absence, a direct neighbor who can progress requests towards destination, use carry and forward method.

b) Switch to radio transmission channel as soon a vehicle moves into the vicinity which can be selected as thenext hop towards the destination

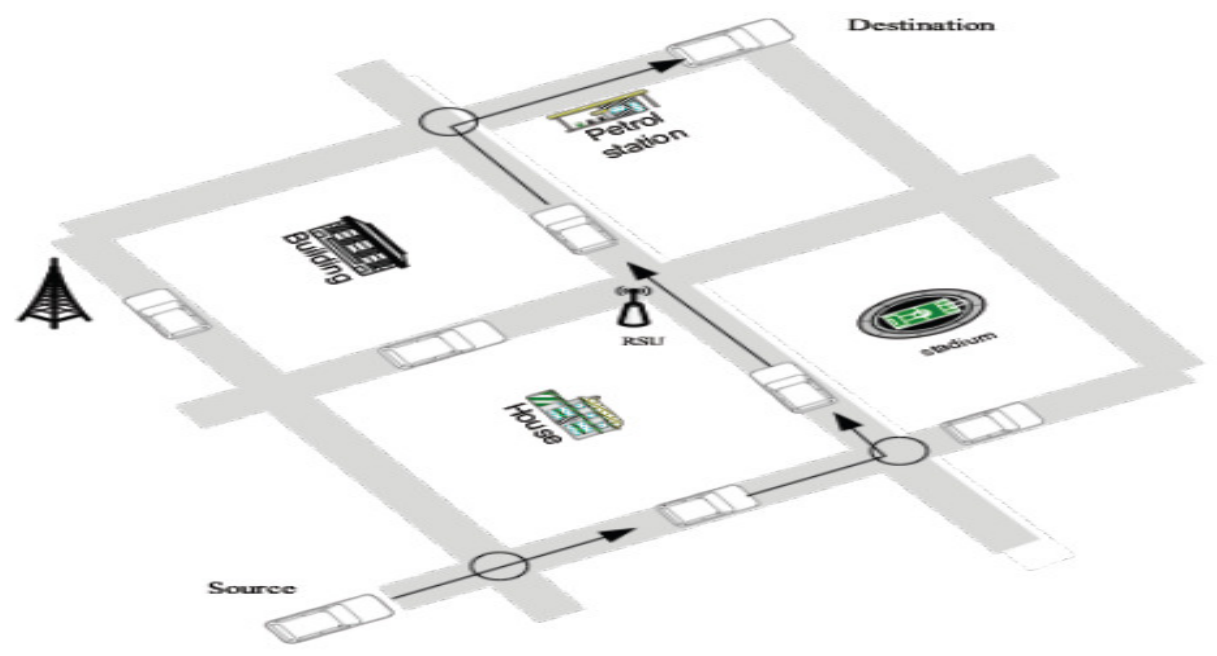

Figure 1. Scenario for Vehicle-to-Vehicle communication 
International Journal of Computer Networks \& Communications (IJCNC) Vol.9, No.1, January 2017

\section{Proposed Routing Algorithm}

\section{Position based Adaptive Routing (PAR) Protocol}

The proposed Position based Adaptive Routing (PAR) protocol is split into four phases:

- Destination location and path discovery.

- Data packet forwarding along the found path.

- $\quad$ Path maintenance.

- $\quad$ Error recovery.

\subsection{Destination Location and Path Discovery}

The source node knows its position through GPS and gets the location and the velocity vector of the destination with the help of a location service. The Algorithm uses one hop beacon messages from surrounding nodes to construct neighbor table at each node. The beaconing internal of nodes in a specific area depends on the amount of vehicle density in that area. The beacon interval is more when network density is high and in case of fewer neighbors it is less. The beacons used by vehicles include its location, moving direction and speed. The source node generates a route request (RREQ). The RREQ packet contains (the) position of source node ( $\mathrm{S}$ _id), its own velocity vector (S_v), TTL for of that packet, destination'sposition (D_id) and destination's velocity vector (D_id). RREQ is sent by using the preferred broadcasting mode by the source. In this mode the source node after broadcasting the route request starts listening to the channel. If the packet is not further rebroadcasted by any neighbor in a set timeout, then it repeats the broadcast. This process is repeated until the request reaches the destination. Any intermediate node $\mathrm{N}$ which is in junction or if its angle with previous node which handed it the packet is $>180$, updates the packet header and records the current geographical position (ties an anchor $I_{i}$ ) in it. So the header of route request (RREQ) packet records all the list of anchor points traversed by it on its way toward the destination.

If intermediate node $\mathrm{N}$ finds no neighbors in itsrange which can progress data delivery towards the destination, then it switches to carry and forward mode until next junction. From junction again node switches back to preferred group broadcasting mode. This process continues until the packet reaches destination D or its TTL becomes zero. On receipt of RREQ destination D waits for preset some time, record more possible Route Requests. D selects the shortest path among those received route requests. It sends the RREP which is a unicast packet to thesource S. RREP contains the destination coordinates, its velocity vector together with theinformation recorded during the route request phase. Advance greedy forwarding is used to send RREP through recorded anchors point to the source.

\subsection{Data Packet Forwarding Along the Found Path}

Once the source node receives RREP packet from the destination, now it has determined a path to reach the destination. Data packets are routed geographically from one anchor node to the next anchor node until they reach the destination. This is done by the source node $\mathrm{S}$ by using source routing approach. The list of anchor nodes to be traversed is included in every transmitted data 
packet by the source. PAR does it by greedily transferring information between two anchor nodes. The authors of CAR [6] used anchor points to work with the Advance Greedy Forwarding (AGF). AGF presumes that velocity vectors are exchanged by the source and the destinations. The intermediate forwards the packet node to a neighbor closest to the next anchor point, instead of forwarding a data packet to a neighbor that is geographically closer to the destination. Each forwarding node relays to anchor if the distance is less than half coverage to avoid multiple attempts to gradually get closer to the next anchor point. Each forwarding node checks if its position and the position of the next anchor point are separated by less than half of the node's coverage range. The process continues until the packet reaches its destination.PAR employs the guards [6] (Shown in Figure 2) to help intermediate node $\mathrm{N}$ to find out if a message has reached a certain anchor node or not. Two types of guards are used i.e., stationary guards and moving guards. A standing guard represents temporary state information that is tied to a geographical area, rather than to a specific node.If two intermediate node's velocity vectors angle is more than $18^{\circ}$, anchor is set $[3,6]$. Anchor contains coordinates and velocity vector of the current node and previous node. The nodes located in the area are kept alive by the guard. The beacon message of a node contains the guard entry.

The new entry contains an id, a TTL counter, a guarded location coordinates and the radius. An intermediate node with a guard can filter or redirect packets or adds information to a packet which is further transmitted to a node geographically near to the destination [6]. Once TTL reaches zero, the guard is removed from the node's HELLO beacon. In the event of the intermediate not finding any neighbor, the algorithm switches to carry and forward mode [5] and carries the packet. Based on the existing traffic pattern, a vehicle can find the next road to forward the packet to reduce the delay $[5,8]$.

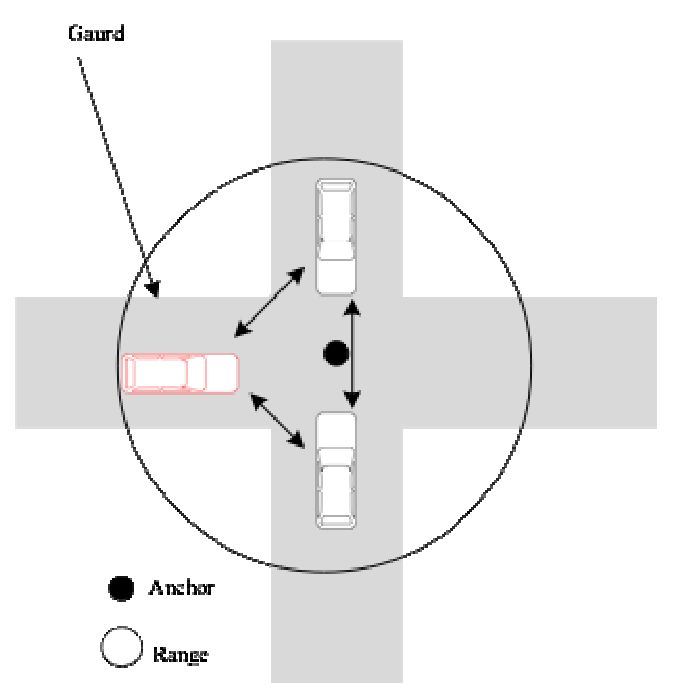

Figure 2. Guard being exchanged by vehicles 
The VADDmodel proposed by the authors [5] share the idea of storing and forwarding the data packet. In the VADD the main goal is to select the path with the smallest packet delay. The delay model used in VADD is shown in Figure 3. A vehicle located near junction $\mathrm{I}_{\mathrm{a}}$ computes the delay for the road between $I_{a}$ and $I_{b}\left(D_{a b}\right)$ by taking into consideration also the estimated delay of choosing the road between $\mathrm{I}_{\mathrm{b}}$ and $\mathrm{I}_{\mathrm{x}}$, the one between $\mathrm{I}_{\mathrm{b}}$ and $\mathrm{I}_{\mathrm{y}}$ or the one between $\mathrm{I}_{\mathrm{b}}$ and $\mathrm{I}_{\mathrm{z}}$. Once the next road has been selected, next is to choose a relay vehicle towards the destination. PAR algorithm switches back to position based routing when it is able to find a connected path between the source and the destination. The flowchart of the proposed algorithm is shown in Figure 4. The algorithm is described in Figure 5.

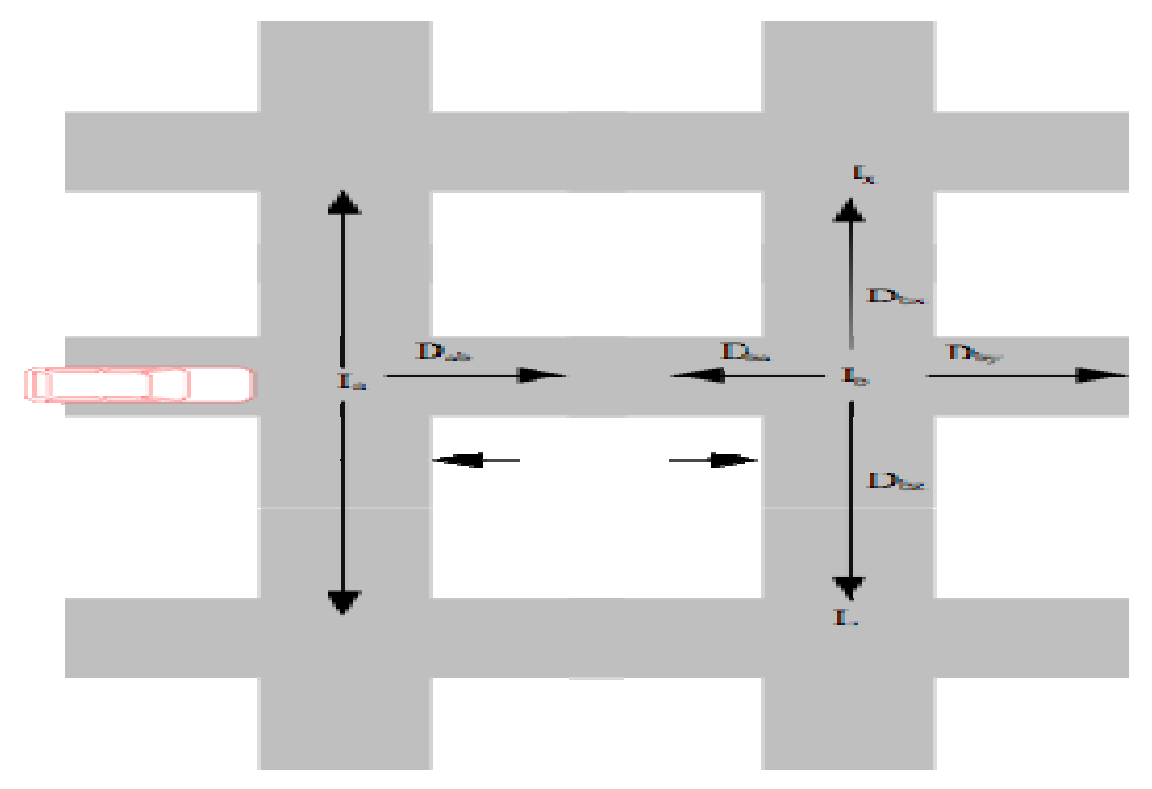

Figure 3. Scenario for VADD model

\subsection{Path Maintenance}

Similar to [3, 6] PAR uses anchor points for data forwarding. If no node is guarding a particular anchor point, the algorithm employs carry and forward strategy. If the destination node is moving and it changes direction then it activates a standing guard. The newly created guard stores the old and the new velocity vectors of the destination. Whenever a node which is guarding a particular location receives a message addressed tothe node that has initiated the guard, the guarding node adds the guarded location as an anchor point to the packet header, updates the new estimated location of the destination, and forwards the packet.Standing guard is attached to a geographical area, rather than to a specific node. A traveling guard is activated if the intermediate or the destination node changes direction against the direction of communication [6].Traveling guard contains position, velocity vector, and radius. A traveling guard runs as the end node's old direction and speed, and routes the packets to the new position of the destination. 
International Journal of Computer Networks \& Communications (IJCNC) Vol.9, No.1, January 2017

\subsection{Error Recovery}

PAR algorithm uses preferred group broadcasting (PGB) to find connected paths between the source and the destination. It uses store and forward technique until next junction if gets stuck up in local maximum [2]. It uses only anchor nodes for data forwarding to the destination. Due to the varying network density, routing errors may still cause problems. A message may reach the estimated destination position but fails to find the node there. This happened as destination node before moving could not activate guard due to lack of neighbors in its radio range.The algorithm in such cases uses store carry and forward method and hands over the packet as soon as it finds any node moving in its vicinity and geographically closer to the destination. In another case, the intermediate node changes its direction from the direction ofthe communicationand does not find any neighbor to activate a guard then it sends an error notification to the source node. Source stops sending any data packet on the current discovered path and starts a new path discovery process.

\section{Performance Evaluation}

To evaluate the proposed routing algorithm, PAR, we have used a simulator based on SUMO [18] for traffic simulation and NS2 [20] for network simulation. We have also simulated GPSR [13], GPCR [2], and CAR [6] for comparison with PAR algorithm.

\subsection{Performance Metrics}

The prime goal of any routing scheme is to increase packet delivery or service ratio and at the same time reduce average packet delay betweenthe source and the destination. A good routing algorithm should also have low message overhead. We use the following metrics for performance evaluation.

- Service ratio (\%). Itis defined as the ratio of the number of packets sent by the source vehicle to the number of packets received by the destination vehicle. A better routing algorithm should have a high service ratio.

- Average delay of a data packet (s). It is the average time taken by the packets from the source vehicle to the destination vehicle. 


\begin{tabular}{|c|c|}
\hline $\begin{array}{l}1 . \\
\text { the st: }\end{array}$ & $\begin{array}{l}\text { Node } \mathrm{S} \text { get destination D location from location server (I have capitalized } \\
\text { ting words) }\end{array}$ \\
\hline 2. & Construct neighbor table from one hop beacon packets \\
\hline $\begin{array}{l}3 . \\
\text { tion }\end{array}$ & Forward the packet to the neighbor that is geographically closer to destina- \\
\hline $\begin{array}{l}4 . \\
\text { go to }\end{array}$ & $\begin{array}{l}\text { If intermediate nodes } \mathrm{N} \text { towards destination is in range i.e. } P_{d}=1-\mathrm{e}^{-p_{\mathrm{i}} \mathrm{T}_{\mathrm{v}}} \\
\text { tep } 6\end{array}$ \\
\hline 5. & Otherwise, carry the packet until next junction \\
\hline 6. & If node $\mathrm{N}$ is in junction or angle between communicating nodes is $>180$ \\
\hline & Set anchors Ii and tie it to that particular location \\
\hline $\begin{array}{l}8 . \\
\text { its TT }\end{array}$ & $\begin{array}{l}\text { Keep forwarding packet to the next node until reaches the destination D or } \\
=0\end{array}$ \\
\hline 9. & Destination D waits for some time record all possible Route Requests \\
\hline 10. & Destination D selects the lowest delay path to send route reply to source \\
\hline 11. & Use the recoded anchors in packet header of Route Reply to transfer data \\
\hline $\begin{array}{l}12 . \\
\text { node }\end{array}$ & Set static guard for moving stationary source, intermediate or destination \\
\hline $\begin{array}{l}13 . \\
\text { comn }\end{array}$ & $\begin{array}{l}\text { Set moving guard for a node which moves opposite to the direction of } \\
\text { nnication }\end{array}$ \\
\hline
\end{tabular}

Figure 4. PAR algorithm

\subsection{Experimental Setup}

The experiment is based on a $2000 \times 2000 \mathrm{~m}^{2}$ square street area in a grid layout. The street layout, roads, mobility pattern and vehicles are generated by using SUMO [18]. The generated mobility trace is transformed into a data format that is used as input to NS2 [20]. To have different network densities, the number of vehicles moving into the area has been varied between 10 and 60 . All these vehicles are moving forward and backward during the simulation to have the continuous traffic flow in the street area. When one vehicle reaches the end of the road, which means the vehicle will move out of the grid area, it no more participates in the network. Various simulation parameters with their corresponding values are listed in Table 1. 
International Journal of Computer Networks \& Communications (IJCNC) Vol.9, No.1, January 2017
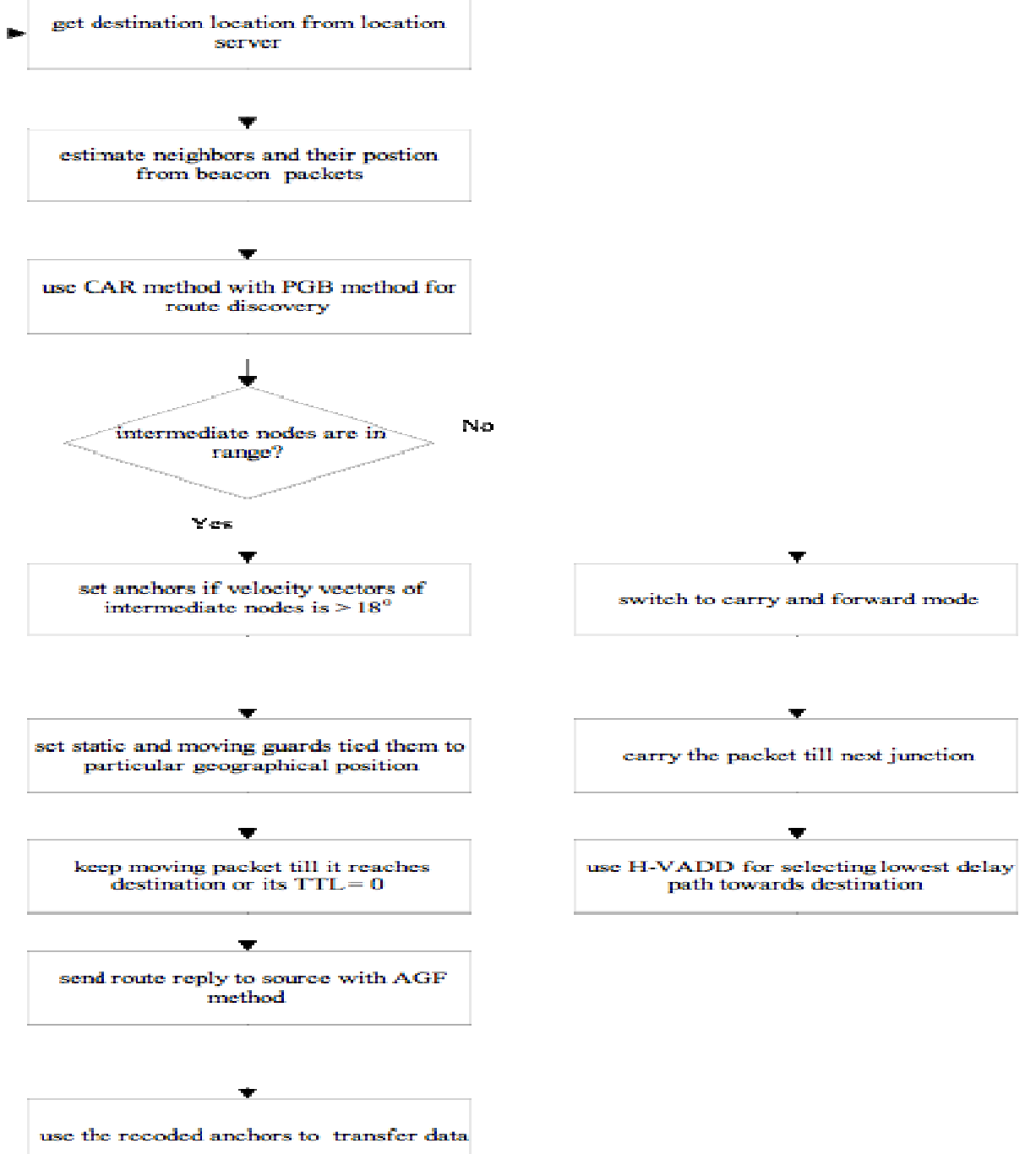

Figure 5. Flowchart of PAR algorithm

Table 1.Simulation Parameters.

\begin{tabular}{|c|c|}
\hline Parameter & Value \\
\hline Simulation Time & $1000 \mathrm{~s}$ \\
\hline Maximum Communication Range & $200 \mathrm{~m}$ \\
\hline Vehicle Speed & $30 \mathrm{~km} / \mathrm{hour}$ \\
\hline Transmission Rate & $11 \mathrm{Mbps}$ \\
\hline
\end{tabular}


International Journal of Computer Networks \& Communications (IJCNC) Vol.9, No.1, January 2017

\begin{tabular}{|c|c|}
\hline Item Size & $10 \mathrm{~B}-4 \mathrm{~KB}$ \\
\hline Inter Vehicle Distance & $20 \mathrm{~m}$ \\
\hline Vehicle Beacon Interval & $0.5 \mathrm{sec}$ \\
\hline Packet TTL & $128 \mathrm{sec}$ \\
\hline
\end{tabular}

We have evaluated GPSR, GPCR, CAR and the proposed algorithm, PAR, and compared the performance.

\subsection{Results}

\section{Effect of Vehicle Density on Service Ratio}

Figure 6 shows the service ratio as a function of vehicle density in the area. The item size is set to 2500 bytes for the experiment. The vehicle densityvaries between minimum 10 vehicles to maximum 60 vehicles in the grid area. For all traffic volumes, GPSR does not perform well as it has a poor delivery ratio. The GPSR does not perform well in this scenario because it uses greedy forwarding for selection of the next hop so more often gets stuck up in local maximum. The GPCR however has a better delivery ratio as compared to GPSR and increaseswith number of vehicles in the area. This uses perimeter coordinator routing for selection of next hop so better service ratio. The CAR protocol uses preferred group broadcasting for route discovery and uses advance greedy forwarding for data forwarding so achieves high service ratio. The service of CAR increases with number of vehicles as chances of void in the network are reduced.

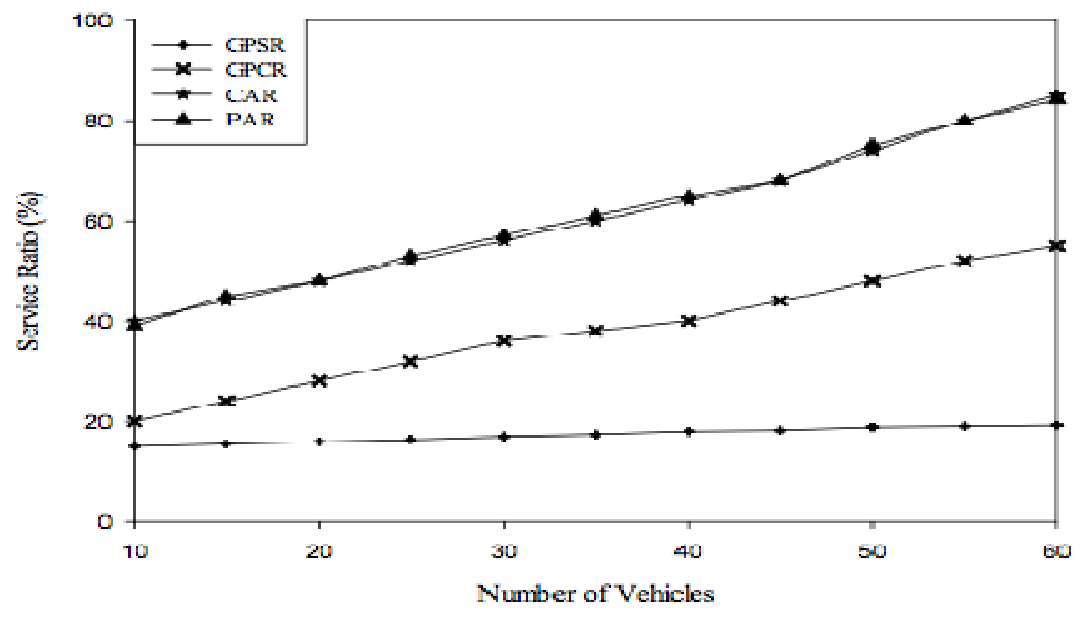

Figure 6. Effect of vehicle density on service ratio

The performance of PAR is almost similar or better than CAR algorithm as the number of vehicles increase from 10 to 60 . The PAR algorithm uses two modes and works similar to CAR [6] in mode one, when a vehicle is not able to find a neighbor to route a packet to the destination and 
switches to VADD [5] mode. Theintermediate vehicle when does not find any other vehicle in its radio range then it carries the packet until the next junction.

\section{Effect of Vehicle Density on Average Packet Delay}

The item size is again set to 2500 bytes for the experiment. The average packet delay for all algorithms gets reduced with the increase in number of vehicles. The reduction is least in case of GPSR and GPCR that use only geographical data for route discovery and data forwarding. These algorithms often fail due to local maximum. With the increase in number of vehicles in the network the chances of finding at least one neighbor for packet forwarding increases. CAR considers current traffic pattern and vehicle density, so have lower packet delay and it further reduces with increase in the number of vehicles as more connected paths are found between the source and the destination. Further CAR easily tolerates short term disconnections due to partitions or temporary interference.

PAR algorithm has the lowest average packet delay because most of the time itis able to find an end to end connected path between the source and the destination. This algorithm switches to buffer and forward mode when partitions are formed in the network. The destination node on receipt of a request does not reply back immediately rather it waits for some time, keeps on accumulating route requests from the source node and selects the minimal path request from these accumulated requests. So PAR algorithm has the auto-adjustability capability to maintain a least delay subpath between the source and the destination and has the lowest average delay as shown in Figure 7.

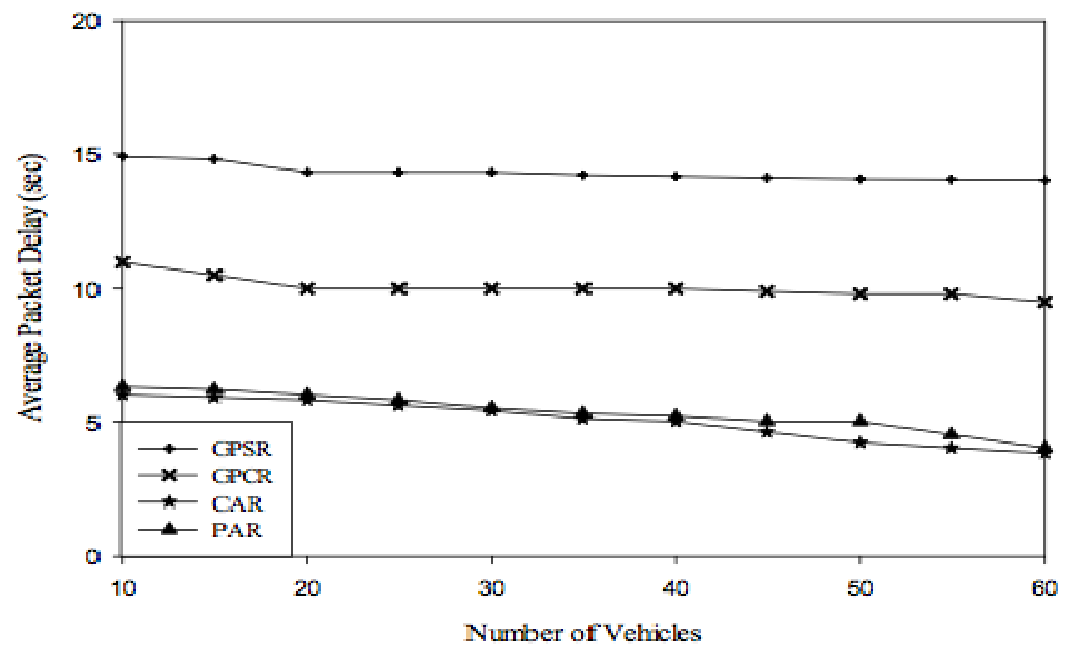

Figure 7. Effect of vehicle density on average packet delay

\section{Effect of Data Packet Size on Service Ratio}

Larger packet size consumes more bandwidth. So as we increase the data packet size from 10 bytes to 4000 bytes the contention for wireless channel increases. In this case we have assumed 
25 vehicles are always available in the grid area. The service ratio drops much faster in case of GPCR as compared to CAR and PAR algorithms. PAR has the least decrease in the service ratio because it does not forward data to the next vehicle which is closer to the destination rather it forwards it to the next junction, so in this case header will have lesser control information as compared to CAR algorithm shown in Figure 8. In case of GPSR the service ratio more or less remain same for all data packet size as it only uses greedy forwarding for route discovery and data forwarding phases. This intermediate node in GPSR more often gets stuck up in local maximum.

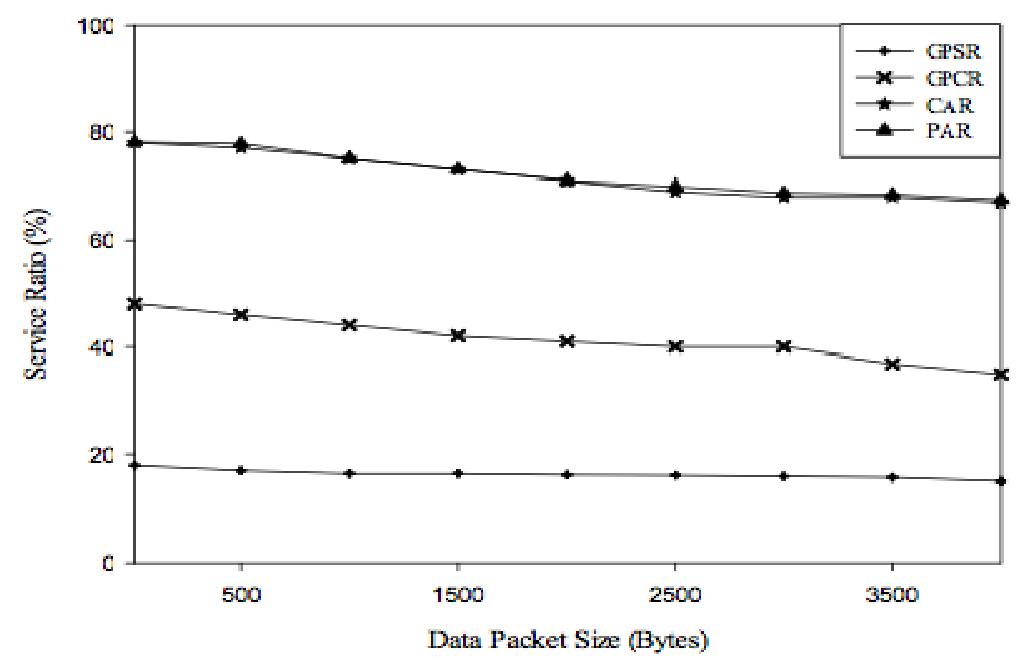

Figure 8.Effect of data packet size on service ratio

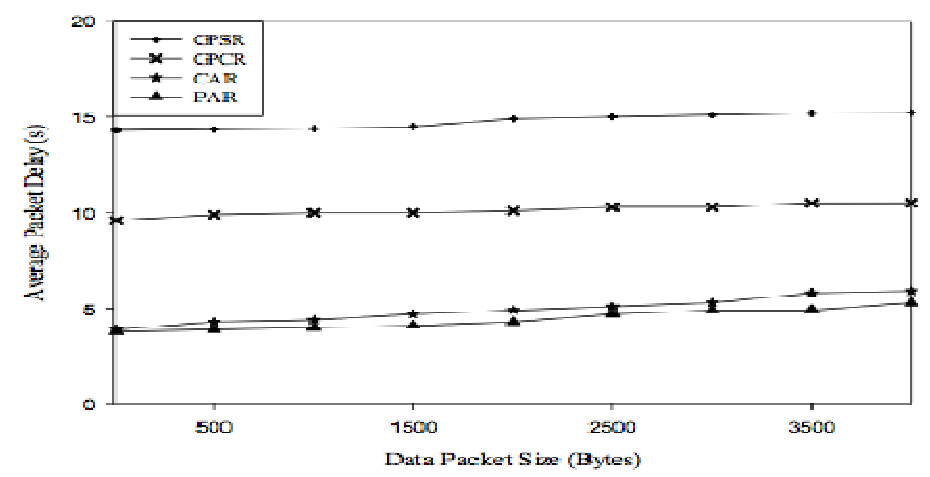

Figure 9. Effect of data packet size on average packet delay

\section{Effect of Data Packet Size on Average Packet Delay}

Effect on data packet size on average packet delay is shown in Figure 9. The average packet delay increases across all compared algorithms as we increase the data packet size from 10 bytes to 
International Journal of Computer Networks \& Communications (IJCNC) Vol.9, No.1, January 2017

4000 bytes. The large data packet size will consume more bandwidth so less fewer wireless channels remains available. So more data packets get dropped as the bit error rate (BER) increases which in turn increases the average packet delay in the network. In this case also we have taken total 25 vehicles for the experiment. The experiment is carried out with a fixed transmission rate. The GPCR observers more increase as compared to CAR and PAR both of them very much similar increase in average packet delay. This pattern is because the packet forwarding is not done hop-by-hop rather it is done junction wise in CAR and PAR.

\section{Conclusions}

In this paper we have proposed a Position based Adaptive Routing (PAR) scheme which is scalable to different densities of vehicular ad hoc networks. This scheme uses preferred group broadcasting for route discovery. In this mode, after broadcasting the request for route discovery the source node starts listening to the channel. If the packet is not further rebroadcasted by any neighbor in a set timeout, then it repeats the broadcast. This process is repeated until the request reaches the destination. The destination keeps on accumulating route requests coming from different paths until apredefined time. It then chooses the least cost path as route reply. It uses the set of traversed anchors for sending the unicast route reply to the source node. PAR uses advance greedy forwarding for data forwarding and greedily forwards the data packet to the next anchor towards destination node. It switches to carry and forward mode once it finds gaps in the network. The node buffers the packet till next junction and switches back to position based scheme and greedily forwards it to the next node in its range which is closest to the destination. To have an end to end connected path, it uses guards to guard anchors tied to the different junction(s) and

geographical locations in the network. The algorithm is scalable and exploits (the) advantages of existing techniques already developed for the specific scenarios in VANET.

\section{REFERENCES}

[1] Z. Haas, J. Halpernand and L. Li, "Gossip Based Ad Hoc Routing," Proceedings of IEEE INFOCOM, pp. 1707-1716, 2002.

[2] C. Lochert, M. Mauve, H. Hartenstein and S. Holger, "Geographic Routing in City Scenarios," ACM SIGMOBILE Mobile Computing and Communications Review, Vol. 9, No. 1, pp. 69-72, 2005.

[3] V. Naumou, R. Baumann and T. Gross, "An Evaluation of Inter-Vehicle Ad Hoc Networks Based on Realistic Vehicular Traces," ACM International Symposium on Mobile Ad Hoc Networking and Computing, Florence, Italy, pp. 108-119, 2006.

[4] M. Jerbi, S. Senouci, M. Meraihi and R. Ghamri, "An Improved Vehicular Ad Hoc Routing Protocol for City Environments," IEEE International Conference on Communications (ICC 2007), Glasgow, Scotland, pp. 3972-3979, 24-28 June 2007.

[5] J. Zhao and C. Cao, "VADD: Vehicle-Assisted Data Delivery in Vehicular Ad Hoc Networks," IEEE International Conference on Computer Communications (INFOCOM 2006), pp. 1-12, 2006.

[6] V. Naumov, and T. Gross, "Connectivity Aware Routing (CAR) in Vehicular Ad Hoc Networks," IEEE International Conference on Computer Communications (INFOCOM 2007), pp. 1919-1927, 2007.

[7] K. Katsaros, M Dianati and K. Roscher, "A Position-Based Routing Module for Simulation of VANETs in ns-3," SIMUTOOLS '12, pp 345-352, 2012.

[8] M. Boban, G. Misek and O. Tonguz, "What is best acheivableQos for Unicast routing in VANETs ?," IEEE GLOBECOM Workshops, pp.1-10, Jan 2008. 
International Journal of Computer Networks \& Communications (IJCNC) Vol.9, No.1, January 2017

[9] S. Wang, "The Effects Of Wireless Transmission Range On Path Lifetime In Vehicle-Formed Mobile Ad-Hoc Networks On Highways," IEEE International Conference on Communications, Vol. 5, pp. 3177-3181, May 2005.

[10] D.E. Cooper, P. Ezhilchelvan and I. Mitrani, "A Family of Encounter-Based Broadcast Protocols for Mobile Ad-Hoc Networks," International Workshop of the EURO-NGI Network of Excellence, pp. 235-248, June 2004.

[11] F. Bonnet, P. Ezhilchelvan and E. Vollset, "Quiescent Consensus in Mobile Ad- Hoc Networks Using Eventually Storage-Free Broadcasts," Annual ACM Symposium on Applied Computing, pp. 670-674, April 2006.

[12] O. Abedi, M. Fathy and J. Taghiloo, "Enhancing AODV Routing Protocol Using Mobility Parameters in VANET," IEEE/ACS International Conference on Computer Systems and Applications, pp. 229235, 2008.

[13] B. Karp and H. T. Kung, "Greedy Perimeter Stateless Routing for Wireless Networks," Proc. of 6th Annual ACM/IEEE Int. Conf. on Mobile Computing, pp. 243-54, Aug 2000.

[14] C. Lochert, H. Hartenstein, J. Tian, H. Fubler, D. Hermann and M. Mauve, "A Routing Strategy for Vehicular Ad Hoc Networks in City Environments," Proc. of IEEE on Intelligent Vehicles Symposium, pp. 156-161, June 2003.

[15] B. Seet, G. Liu, B. Lee, C. Foh, K. Wong and K. Lee, "A-STAR: A Mobile Ad Hoc Routing Strategy for Metropolis Vehicular Communications," Lecture Notes in Computer Science, Vol. 3042, pp. 989999, Jan 2004.

[16] J. Tian, L. Han, K. Rothermel and C. Cesh, "Spatially Aware Packet Routing for Mobile Ad Hoc Inter Vehicle Radio Networks," Proc. of IEEE on Intelligent Transportation systems, Vol. 2, pp. 15461551 , Oct 2003.

[17] A. Vahdat and D. Becker, "Epidemic Routing for Partially Connected Ad Hoc

[18] MOVE (Mobility model generator for vehicular networks), http://lens.csie.ncku.edu.tw/.

[19] NoW (Network on Wheels), http://www.network-on-wheels.de.

[20] NS2 (the Network Simulator), http://www.isi.edu/nsmam/ns/. 